\title{
Dinámicas socio-espaciales y previsiones demográficas en la cuenca internacional del río Miño-Sil ${ }^{1}$
}

\author{
Julián MORA ALISEDA \\ Departamento de Ciencias del Territorio \\ Universidad de Extremadura \\ jmora@unex.es \\ Jacinto GARRIDO VELARDE \\ Departamento de Ciencias del Territorio \\ Universidad de Extremadura \\ jgvelarde@unex.es \\ Manuel DíAZ GonZÁLEZ \\ Departamento de Ciencias del Territorio \\ Universidad de Extremadura \\ manudiaz.geo@gmail.com
}

Recibido: 29 de noviembre de 2014

Enviado a evaluar: 1 de diciembre de 2014

Aceptado: 27 de abril de 2015

\section{RESUMEN}

En las últimas décadas, la distribución y redistribución de la población sobre el territorio ha cobrado importancia como temática de interés social tanto a escala nacional como regional. El presente trabajo se inserta en los estudios que analizan los cambios ocurridos en el sistema de asentamiento de la población y su efecto sobre la organización espacial de la red urbana. El objetivo de esta investigación es analizar los cambios acaecidos en la distribución de la población de los municipios ubicados en la cuenca internacional del río Miño-Sil.Se trata de una pesquisa de tipo cuantitativa, en su abordaje metodológico, a través de la utilización de datos censales de población considerando el período 2001-2011. En el estudio de los municipios localizados en la cuenca internacional del río Miño-Sil se aplicarán categorías de tamaño de población. Asimismo, se examinará la estructura de la población actual y se realizará un modelo predictivo de la evolución de la población con el fin de podernos anticipar racionalmente a las tendencias futuras de las poblaciones analizadas.

Palabras clave: Índice de ruralidad, dinámicas socio-espaciales, modelos predictivos.

\footnotetext{
${ }^{1}$ Esta investigación ha sido financiada por el Ministerio de Economía y Competitividad a través del proyecto de investigación titulado "Planificación estratégica y gestión compartida de los recursos hídricos hispano-lusos” (Ref CGL2010-19311). Investigador principal: Julián Mora Aliseda.
} 


\title{
Socio-spatial dynamics and demographic estimates in the basin international Miño-Sil River
}

\begin{abstract}
In the last decades, distribution and redistribution of the population on the territory has become important as a subject of social interest both regional and national scale. This work is inserted in studies analyzing changes in the settlement system of the population and its effect on the spatial organization of the urban network. The objective of this research is to analyze the changes in the distribution of the population of the municipalities located in the basin international Miño-Sil River. This is an investigation of quantitative type, in its methodological approach, through the use of census data of population considering the period 2001-2011. In the study of the municipalities located in the basin Miño-Sil population size categories were applied. Also, the structure of the current population is examined and a predictive model of the evolution of the population in order of being able to rationally anticipate future trends in the populations analyzed were performed.
\end{abstract}

Key words: Rurality index, socio-spatial dynamics, predictive models.

\section{Dynamiques socio-spatiales et les prévisions démographiques par le bassin du fleuve international Miño-Sil}

\section{RÉSUMÉ}

$\mathrm{Au}$ cours des dernières décennies, la distribution et la redistribution de la population sur le territoire a pris de l'importance comme un sujet d'intérêt social à la fois l'échelle régionale et nacional. Ce travail fait partie des études portant sur les changements dans le système de règlement de la population et son effet sur l'organisation spatiale du réseau urbain. L'objectif de cette recherche est d'analyser les changements dans la répartition de la population des municipalités dans le bassin international de la rivière Miño-Sil. Ce est une enquête de type quantitatif, que l'approche méthodologique, grâce à l'utilisation des données de population du recensement en tenant compte de la période 2001-2011. Dans l'étude des municipalités situées dans les Miño-Sil catégories internationales de taille de la population du bassin se appliquer. En outre, la structure de la population actuelle est examinée et un modèle prédictif de l'évolution de la population d'être en mesure d'anticiper les tendances futures rationnelle dans les populations analysées ont été effectuées.

Mots clés: Indice de la ruralité, la dynamique socio-spatiales, des modèles prédictifs.

\section{INTRODUCCIÓN}

El conocimiento de los efectivos y de las características de la población ha sido uno de los objetivos perseguidos por los Estados desde la antigüedad a nuestros días, debido a intereses militares y económicos.

El comportamiento de la población es un dato básico a tener en cuenta en cualquiera de los estudios a considerar y en los posibles escenarios que se quieran representar desde una perspectiva de futuro. Por consiguiente, al evaluar el presente 
para actuar en el futuro, es necesario tener suficiente información sobre la población, tanto para observar el comportamiento y satisfacción real de la demanda social como para diseñar una adecuada política económica y social que responda a las necesidades básicas de la población que ocupa y consume el espacio (Requés y Rodríguez, 1996).

El acelerado proceso de urbanización a escala global en las últimas décadas, ha dado lugar a la consolidación de un modelo de concentración desigual, es decir, una heterogénea distribución de la población sobre el espacio geográfico, generando desequilibrios territoriales (Naciones Unidas, 2008; Prieto, 2011). La intensidad espacial y la rapidez de los cambios recientes en España y Portugal ofrece un ejemplo paradigmático del rápido cambio hacia un nuevo sistema de relaciones rurales urbanas de la cuenca internacional del río Miño-Sil, basada en una dicotomía entre las zonas urbanas y rurales (Stellmes et al., 2013). Un profundo conocimiento de los principales cambios espaciales puede mejorar la gestión futura con el objetivo de mitigar la degradación del suelo y su fragmentación, la despoblación, la pérdida de recursos naturales y para reequilibrar las presiones humanas excesivas en las zonas urbanas (Serra et al., 2014). En este sentido, nuestra investigación se engloba en los estudios que analizan los cambios recientes ocurridos en el sistema de asentamiento de la población y su efecto sobre la organización espacial de la red urbana de la cuenca del Miño-Sil hispano-luso.

El objetivo principal, por tanto, es determinar los flujos poblaciones en los municipios hispano-lusos de la cuenca internacional del río Miño-Sil. Este objetivo se complementa con otros más específicos, que se son:

a) Averiguar el índice de ruralidad actual de la cuenca;

b) Evaluar la evolución actual de la población (2001-2011);

c) Analizar la estructura de la población;

d) Elaborar un modelo predictivo de la evolución de la población a partir de análisis de la población actual.

\subsection{MARCO TEÓRICO}

El concepto de ruralidad y mundo rural es objeto de debate científico sin que haya unanimidad al respecto. La definición de zona rural establecida por la Organización para la Cooperación y el Desarrollo Económicos (OCDE) se basa en una combinación entre el tamaño del territorio y la densidad de población. El territorio puede ser la unidad administrativa que corresponda al municipio (en términos de la UE / Eurostat NUTS-Framework: LAU 1). LAU 1 se considera rural, si la densidad de población es de menos de 150 habitantes por kilómetro cuadrado. La definición de la OCDE en la mayoría de los países es demasiado amplia para la aplicación práctica, por ello, cada país realiza sus propios ajustes (Johansen and Nielsen, 2012).

Es necesario romper el estrecho paradigma económico en el que se ha situado el papel del sector rural, y trasladarlo al contexto de la política y las instituciones (Pérez, 2001). El medio rural es entonces una entidad socioeconómica en un espacio 
geográfico con cuatro componentes: Territorio, Población, Conjunto de asentamientos y Conjunto de instituciones públicas y privadas (Muñoz, 2012). Parece evidente, que ante tantas definiciones parciales y, a veces, contrapuestas, la dificultad para diferenciar lo rural de lo urbano, tanto cuantitativa como cualitativamente, es casi más intuitiva que discursiva, según se desprende la revisión bibliográfica internacional. (Brennan et al., 2008; Svendsen y Sørensen, 2007; Lee et al., 2005; Bridger y Luloff, 1999; Braden y Mayo, 1999)

La realidad de las zonas rurales de Europa exhibe numerosos e importantes desequilibrios territoriales de diferentes tipos y niveles (EC, 2010a). El carácter permanente de estas disparidades regionales se ha incrementado desde la reciente ampliación de la UE, y la necesidad de avanzar hacia la convergencia territorial, propiciando la inclusión del principio de cohesión territorial en los objetivos de la UE (art. 158 del Tratado de Lisboa). Como resultado, la cohesión territorial se ha convertido en un tema relevante en las políticas de la UE, y una prioridad en las propuestas legislativas de las políticas de cohesión para el período 2014-2020 (EC, 2011) (Sanchez-Zamora et al., 2014).

En este contexto de disparidades territoriales, es evidente que las zonas rurales europeas no evolucionan de forma homogénea, lo que confirma la existencia de diversas dinámicas espaciales. El concepto de las dinámicas territoriales rurales se refiere a los procesos de desarrollo en la estructura socio-económica, el marco institucional, el capital medioambiental de las zonas rurales, y los cambios que acompañan a los efectos del desarrollo (Rimisp, 2007). En este sentido, se podría decir que hay dos tipos de zonas rurales que coexisten dentro de la UE: las que tienen las dinámicas de desarrollo definidas por el fuerte crecimiento económico, la cohesión social y la sostenibilidad ambiental, y aquellas en las que el estancamiento económico, la despoblación y la degradación del medio ambiente persisten (SánchezZamora et al., 2014).

En los últimos años, el aumento de la intensidad de los cambios y la diversidad de las dinámicas que se están produciendo en las zonas rurales han puesto de relieve la necesidad de realizar investigaciones que pueden hacer frente a los desafíos intelectuales y políticos que surgen como resultado de estas cuestiones. Como consecuencia, varios proyectos han tratado de distinguir entre zonas rurales "líderes" y "rezagadas" con el fin de comparar sus características y determinar los factores que podrían explicar estos diferentes procesos de cambio (Sanchez-Zamora et al., 2014). Algunos de los proyectos más importantes de Europa incluyen el Estudio sobre el Empleo, el Crecimiento y la Innovación en las Zonas Rurales (SEGIRA) (EC, 2010b), Oportunidades Europeas de Desarrollo para las Zonas Rurales (EDORA) (Copus et al., 2011), y las Dinámica en las Áreas Rurales (DORAS) (Bryden y Hart, 2001). Más allá de las fronteras europeas, uno de los proyectos más importantes en América Latina es el Proyecto de Dinámicas Territoriales Rurales (Rimisp, 2007). En esta misma línea, varios autores han tratado de identificar los factores claves que influyen en las dinámicas del desarrollo territorial desde una perspectiva económica (Terluin de 2003 y Agarwal et al., 2009), social (Putnam, 1993, Whiteley, 2000, Nelson y 
Sampat, 2001 y Kahila et al., 2009) y la perspectiva ambiental (Hoggart et al., 1995, Courtney et al., 2006 y Langlais y Tepecik Dis, 2009).

\section{MATERIALES Y MÉTODOS}

\section{1. ÁREA DE ESTUDIO}

El río Miño-Sil tiene una longitud de $343 \mathrm{~km}$, desde su nacimiento hasta su desembocadura en el Océano Atlántico entre las ciudades de A Guarda (España) y Caminha (Portugal), por lo que los últimos $76 \mathrm{~km}$ sirven de frontera entre España y Portugal, siendo, además, los $33 \mathrm{~km}$ finales navegables (CONFEDERACIÓN HIDROGRAFICA DEL MIÑO-SIL 2014).

La mayor parte de la cuenca del río Miño-Sil se sitúa en España, en concreto un $95 \%$ de los $17.080 \mathrm{~km}^{2}$ totales, mientras que la parte portuguesa abarca $799 \mathrm{~km}^{2}$ en el noroeste de Portugal, concretamente entre las coordenadas $41^{\circ} 45^{\prime}$ y $43^{\circ} 40^{\prime}$ de latitud $\mathrm{N}$ y $6^{\circ} 10^{\prime}$ y $8^{\circ} 55^{\prime}$ de longitud W. (Figura 1 ).

Figura 1. Mapa de localización de la cuenca hidrográfica internacional del río Miño-Sil.

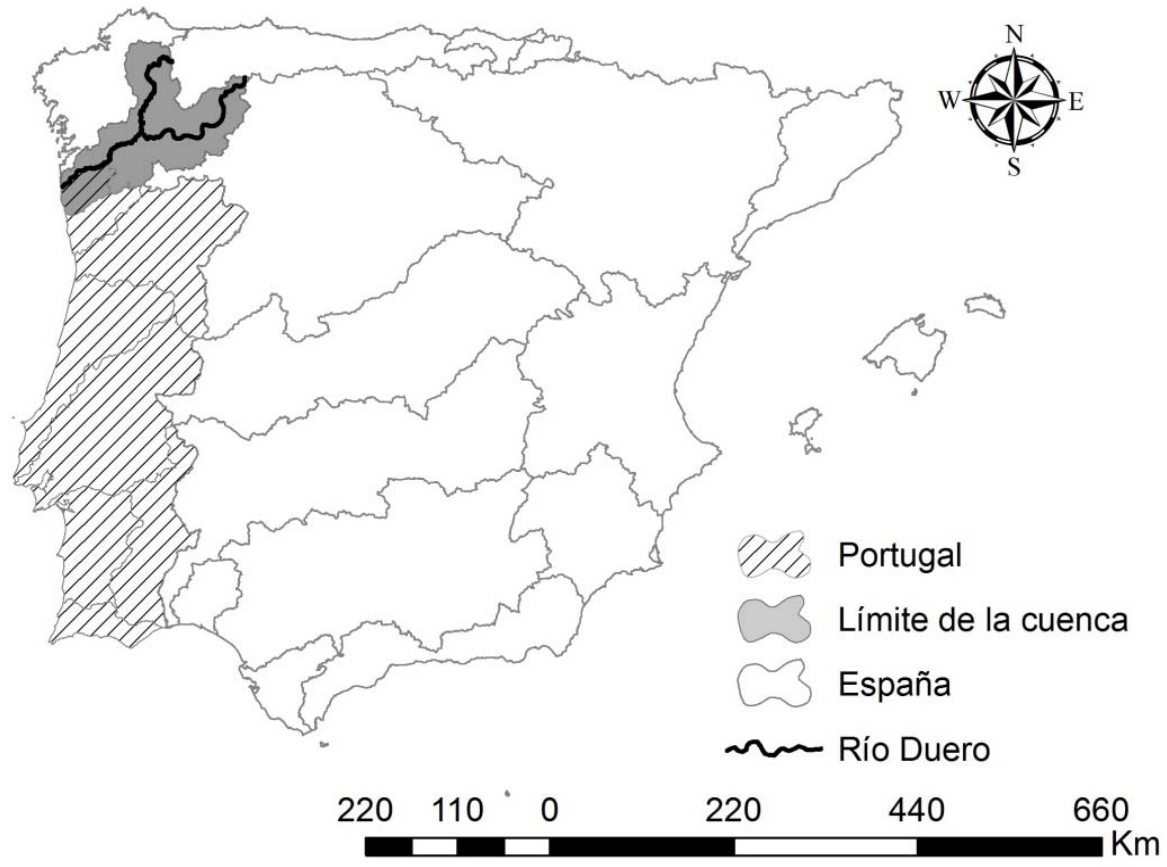

Fuente: Elaboración propia a partir de los dados del CNIG (Centro nacional de Información Geográfica). 


\subsection{METODOLOGÍA DE TRABAJO}

El abordaje metodológico de la presente investigación es de tipo cuantitativo. Se realiza un análisis de la distribución espacial de la población ubicada en la cuenca hidrográfica internacional del río Miño-Sil. A partir de los datos censales se intenta reproducir la conformación del sistema de poblamiento del último censo demográfico (2011).

Una de las formas de presentación de los resultados de los datos censales municipales, consiste en el agrupamiento de las localidades según su tamaño poblacional (Prieto, 2011). En este sentido se definieron 5 estratos de población, logrando así una representación de todos los municipios ubicados en la cuenca internacional del río Miño-Sil: menos de 500; 501 a 2000; 2001 a 5000; 5001 a 10000 y más de 10000 habitantes.

Para el presente estudio también se analizará la evolución de la población actual en la cuenca internacional del río Miño-Sil para el periodo comprendido entre 2001-2011. Para ello, se utiliza como fuente de datos principal los censos nacionales de población de España y Portugal. Teniendo en cuenta las recomendaciones efectuadas por Lindenboim y Kennedy (2003) se trabajará con datos revisados y por lo tanto definitivos.

Con el fin de conocer las tendencias futuras de esa población se realizará un modelo predictivo de la evolución demográfica teniendo en cuenta las variables demografías y el envejecimiento de la población actuales en la cuenca internacional del río Miño-Sil (Requés y Rodríguez, 1996).

El estudio se complementa con la aplicación de la base de datos en un Sistema de Información Geográfica (SIG) con el objeto de establecer la dinámica tanto espacial como temporal y su materialización en la cuenca internacional del río Miño-Sil (López y Pérez 2005; Prieto, 2011).

\section{RESULTADOS Y DISCUSIÓN}

\subsection{DIMENSIÓN POBLACIONAL POR MUNICIPIOS}

En el conjunto de la cuenca la mayoría de los municipios son de pequeño tamaño, ya que el $51,31 \%$ de ellos tiene una población inferior a los 2.000 habitantes. Por el contrario, los municipios con más población $(>10.000)$ representan el $12,04 \%$ del total (Tabla 1). 
Tabla 1. Porcentaje del número de municipios de la cuenca internacional del río Miño-Sil ${ }^{2}$.

\begin{tabular}{|c|c|c|}
\hline Población & $\mathbf{N}^{\mathbf{0}}$ de Municipios & $\%$ \\
\hline Menos de 500 & 13 & $6,81 \%$ \\
\hline $501-2.000$ & 85 & $44,50 \%$ \\
\hline $2.001-5.000$ & 50 & $26,18 \%$ \\
\hline $5.001-10.000$ & 20 & $10,47 \%$ \\
\hline Más de 10.000 & 23 & $12,04 \%$ \\
\hline Total & 191 & $100 \%$ \\
\hline
\end{tabular}

Fuente: Elaboración propia, a partir de los dados del INE-ES-PT. 2001-2011.

\subsubsection{AGRUPACIÓN DE LOCALIDADES POR SU TAMAÑO POBLACIONAL UBICADOS EN ESPAÑA}

La cuenca del Miño-Sil congrega 191 municipios a ambos lados de la frontera, de los cuales 181 pertenecen a la demarcación española y 10 a la portuguesa. La mayoría de los municipios de la parte española son de pequeño tamaño, pues el 54,14\% (98 municipios) tienen menos de 2.000 habitantes. Estos núcleos poblacionales se concentran en el suroeste de la cuenca, en las provincias de Ourense y León, aunque en la provincia de León predominan las localidades con una población inferior a los 500 habitantes (Tabla 2).

Por el contrario, las localidades con más población ( $>10.000$ habitantes) son las que menos representatividad tienen, pues tan sólo constituyen el 8,84\% del total (16 municipios) (Tabla 2). Estos núcleos poblacionales son sobre todo capitales de provincia y sus poblaciones adyacentes como Lugo y Orense.

${ }^{2}$ Agrupados en 5 categorías (menos de 500; 501 a 2000; 2001 a 5000; 5001 a 10000 y más de 10000 habitantes) 
Tabla 2. Porcentaje del número de municipios de la cuenca internacional del río Miño-Sil ${ }^{3}$.

\begin{tabular}{|c|c|c|}
\hline Población & $\mathbf{N}^{\mathbf{0}}$ de Municipios & $\%$ \\
\hline Menos de 500 & 13 & $7,18 \%$ \\
\hline $501-2.000$ & 85 & $46,96 \%$ \\
\hline $2.001-5.000$ & 50 & $27,62 \%$ \\
\hline $5.001-10.000$ & 17 & $9,39 \%$ \\
\hline Más de 10.000 & 16 & $8,84 \%$ \\
\hline Total & 181 & $100 \%$ \\
\hline
\end{tabular}

Fuente: Elaboración propia, a partir de los dados del INE-ES-PT. 2001-2011.

\subsubsection{AGRUPACIÓN DE LOCALIDADES POR SU TAMAÑO POBLACIONAL UBICADOS EN PORTUGAL}

En la demarcación portuguesa encontramos a 10 de los 191 municipios que engloba el sistema urbano del río Miño-Sil, lo que representa el 5,24\% del total. Las localidades portuguesas se caracterizan al contrario que las españolas porque la mayoría de municipios tiene una población mayor a 10.000 habitantes, por lo que constituyen el $70 \%$ del total, seguido muy de cerca por las localidades con una población que varía entre los 5.001 y 10.000 habitantes y que representan el $30 \%$ del total (Tabla 3)

Sin embargo, es preciso señalar que en la demarcación portuguesa no hay ninguna localidad con menos de 5.000 habitantes, todo lo contrario de lo que ocurre en la parte española donde estos municipios constituyen el $81,77 \%$ del total (Tabla 2). No obstante, cabe aclarar que el sistema de poblamiento portugués es muy diferente al español, pues allá el municipio está integrado por numerosas entidades locales menores, denominadas Freguesias, siendo muchas inferiores a 2000 habitantes.

${ }^{3}$ Agrupados en 5 categorías (menos de 500; 501 a 2000; 2001 a 5000; 5001 a 10000 y más de 10000 habitantes) 
Tabla 3. Porcentaje del número de municipios de la cuenca internacional del río Miño-Sil ${ }^{4}$.

\begin{tabular}{|c|c|c|}
\hline Población & $\mathbf{N}^{\mathbf{0}}$ de Municipios & $\%$ \\
\hline Menos de 500 & 0 & 0 \\
\hline $501-2.000$ & 0 & 0 \\
\hline $2.001-5.000$ & 0 & 0 \\
\hline $5.001-10.000$ & 3 & $30 \%$ \\
\hline Más de 10.000 & 7 & $70 \%$ \\
\hline Total & 10 & $100 \%$ \\
\hline
\end{tabular}

Fuente: Elaboración propia, a partir de los dados del INE-ES-PT. 2001-2011.

\subsection{DIMENSIÓN POBLACIONAL POR NÚMERO DE HABITANTES}

En la cuenca internacional del río Miño-Sil viven un total de 1.084 .636 personas, de las cuales 839.800 habitantes $(77,43 \%$ del total) residen en la parte española y $244.172(22,57 \%$ del total) en la parte portuguesa. En el conjunto de la cuenca 686.531 personas $(63,30 \%)$ se asientan en municipios de más de 10.000 habitantes, mientras que tan sólo 110.089 personas $(10,15 \%)$ viven en localidades con menos de 2.000 habitantes.

${ }^{4}$ Agrupados en 5 categorías (menos de 500; 501 a 2000; 2001 a 5000; 5001 a 10000 y más de 10000 habitantes) 
Figura 2. Mapa representativo de la distribución espacial de los municipios por número de habitantes.

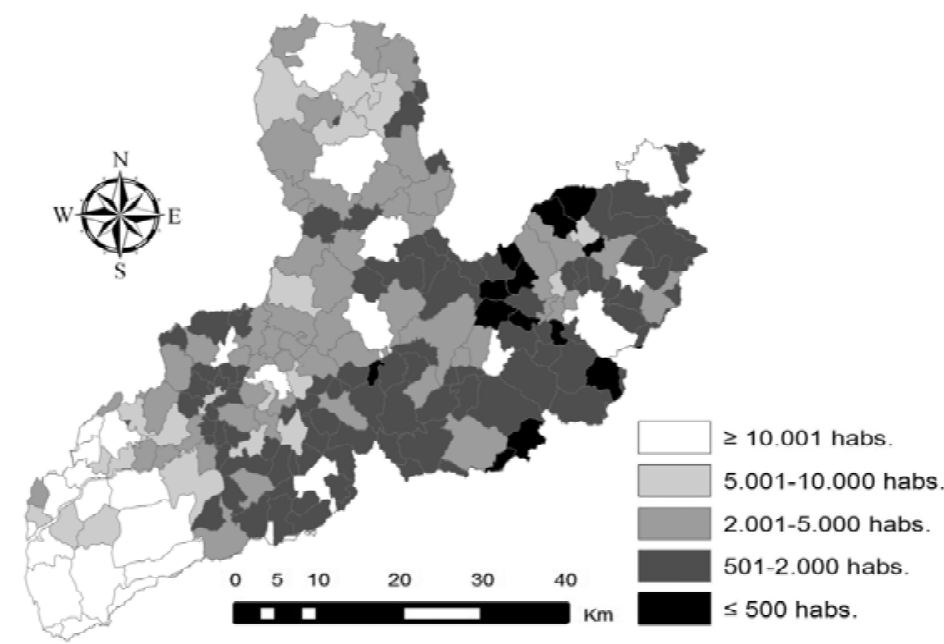

Fuente: Elaboración propia, a partir de los dados del INE-ES-PT. 2001-2011 y los dados del CNIG (Centro nacional de Información Geográfica).

\subsubsection{DIMENSIÓN POBLACIONAL POR NÚMERO DE HABITANTES UBICADOS EN ESPAÑA}

En la demarcación española reside el $77,43 \%$ del total de población de la cuenca (839.800 habitantes). La mayoría de la población está concentrada en poblaciones con más de 10.000 habitantes, ya que representan el 55,89\% del total con 469.359 habitantes. Las tres poblaciones más grandes son Ourense, Lugo y Ponferrada reuniendo a una población de 247.517 habitantes, lo que corresponde al $29,47 \%$ de la población residente en la parte española del Miño-Sil.

Por otra parte, la población que vive en localidades que oscilan entre los 5.001 y los 10.000 habitantes representa el $13,16 \%$ del total. A este le siguen los 149.818 habitantes $(17,84 \%$ del total) que viven en municipios con una población que varía entre los 2.001 y 5.000 habitantes. Por último están las localidades inferiores a los 2000 habitantes, que reúnen a una población total de 110.089 personal y que representan el porcentaje más bajo de la demarcación española con el 13,11 \% (Tabla 4) 
Tabla 4. Porcentaje del número de habitantes que vive en poblaciones en la demarcación española 5 .

\begin{tabular}{|c|c|c|}
\hline Población & Población & \% \\
\hline Menos de 500 & 4.201 & $0,50 \%$ \\
\hline $501-2.000$ & 105.888 & $12,61 \%$ \\
\hline $2.001-5.000$ & 149.818 & $17,84 \%$ \\
\hline $5.001-10.000$ & 110.534 & $13,16 \%$ \\
\hline Más de 10.000 & 469.359 & $55,89 \%$ \\
\hline Total & 839.800 & $100 \%$ \\
\hline
\end{tabular}

Fuente: Elaboración propia a partir de los dados del INE-ES-PT. 2001-2011.

\subsubsection{DIMENSIÓN POBLACIONAL POR NÚMERO DE HABITANTES UBICADOS EN PORTUGAL}

En la demarcación portuguesa ocurre algo similar a la española, pero con mayor porcentaje de población urbana, ya que el $88,70 \%$ de la población (217.172 habitantes) está concentrada en los núcleos mayores de 10.000 habitantes. Las tres ciudades más grandes son Viana do Castelo, Ponte de Lima y Arcos de Valdevez reuniendo entre ellas una población de 150.070 habitantes, lo que supone el 63,34\% del total de la demarcación (Tabla 5).

Por el contrario, en las localidades que oscilan entre los 5.001 y 10.000 habitantes se asientan 27.664 personas, lo que representa el $11,30 \%$ del total. Hay que destacar que en la parte portuguesa no hay ninguna localidad que tenga menos de 5.000 habitantes, como sí ocurre en la parte española (Tabla 4). No obstante, como se comentó en el apartado anterior, cabe aclarar que el sistema de poblamiento portugués es muy diferente al español, pues allá el municipio está integrado por numerosas entidades locales menores, denominadas Freguesias, siendo muchas inferiores a 2000 habitantes.

${ }^{5}$ Agrupadas en 5 categorías (menos de 500; 501 a 2000; 2001 a 5000; 5001 a 10000 y más de 10000 habitantes). 
Tabla 5. Porcentaje del número de habitantes que vive en poblaciones en la demarcación portuguesa ${ }^{6}$.

\begin{tabular}{|c|c|c|}
\hline Población & Población & $\%$ \\
\hline Menos de 500 & 0 & 0 \\
\hline $501-2.000$ & 0 & 0 \\
\hline $2.001-5.000$ & 0 & $11,30 \%$ \\
\hline $5.001-10.000$ & 27.664 & $88,70 \%$ \\
\hline Más de 10.000 & 217.172 & $100 \%$ \\
\hline Total & 244.836 & \\
\hline
\end{tabular}

Fuente: Elaboración propia, a partir de los dados del INE-ES-PT. 2001-2011.

\section{3. ÍNDICE DE RURALIDAD}

En el último siglo se han creado diferentes índices de ruralidad de acuerdo con las condiciones de cada país (Zamudio et al., 2008). El Ministerio de Agricultura, Pesca y Alimentación del Gobierno de España, establece como rural dominante los municipios con menos de 2000 habitantes, rural intermedio los municipios que estén entre 2000 y 10000 habitantes y urbano núcleos con más de 10000 habitantes.

Para el presente estudio se establece como rural dominante los municipios con menos de 5000 habitantes, porque entendemos que se ajusta más al modelo tradicional de asentamientos en España y a la Ley de Bases de Régimen Local ${ }^{7}$, rural

\footnotetext{
${ }^{6}$ Agrupadas en 5 categorías (menos de 500; 501 a 2000; 2001 a 5000; 5001 a 10000 y más de 10000 habitantes)

${ }^{7}$ La Ley 7/1985, de 2 de abril, reguladora de las Bases del Régimen Local, en su art ${ }^{\circ} .26$ señala: 1. Los Municipios por sí o asociados deberán prestar, en todo caso, los servicios siguientes: a) En todos los Municipios: Alumbrado público, cementerio, recogida de residuos, limpieza viaria, abastecimiento domiciliario de agua potable, alcantarillado, acceso a los núcleos de población, pavimentación de las vías públicas y control de alimentos y bebidas. b) En los Municipios con población superior a 5.000 habitantes, además:Parque público, biblioteca pública, mercado y tratamiento de residuos.
} 
intermedio los municipios que estén entre 5000 y 10000 habitantes, que suelen contar con un mayor número de equipamientos y urbano los municipios con más de 10000 habitantes. Este ajuste en los parámetros establecidos se realiza con el fin de adecuarlos a una mejor representación de la realidad de los municipios ubicados dentro de la cuenca hidrográfica internacional del río Miño-Sil.

Los municipios que integran la cuenca internacional del río Miño-Sil tienen un índice de ruralidad predominante; no obstante, hay una clara diferencia a ambos lados de la frontera, ya que mientras en la zona española predomina el índice rural en la portuguesa destaca el índice urbano.

La demarcación española es la que más núcleos poblacionales tiene con un índice rural, pues el $81,76 \%$ de las localidades tienen una población inferior a los 5.000 habitantes. Por el contrario, en la demarcación portuguesa no existe ningún municipio con índice rural (Figura 3).

Figura 3. Mapa del índice de ruralidad de las poblaciones ubicadas en la cuenca internacional del río Miño-Sil.

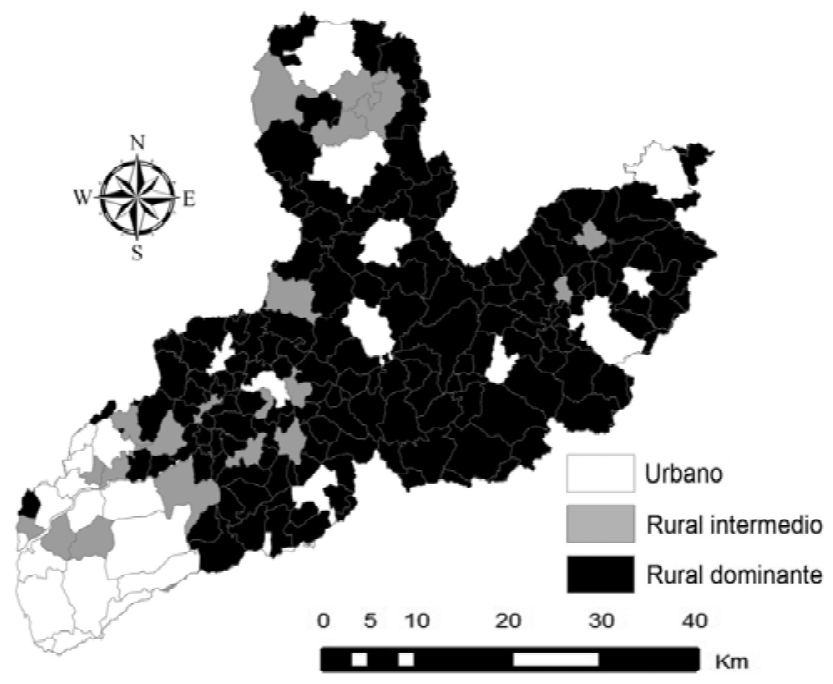

Fuente: Elaboración propia, a partir de los dados del INE-ES-PT. 2001-2011 y los dados del CNIG (Centro nacional de Información Geográfica).

En las zonas donde más destaca el índice rural intermedio es en la demarcación portuguesa, donde el $30 \%$ de los municipios tienen una población comprendida entre los 5.001 y 10.000 habitantes. La mayoría de estas localidades están situadas junto a la frontera española. Por el contrario, en la demarcación española solo en el 9,39\% de los municipios predomina el índice rural intermedio. 
Por último, los municipios con un índice urbano son aquellos que tienen más de 10.000 habitantes. Estas localidades tienen un peso más importante en la parte portuguesa, donde el $70 \%$ (7 municipios) tienen este índice, destacando municipios como Viana do Castelo, Arcos de Valdevez o Ponte de Lima. En cambio, en la demarcación española sólo el 8,84 \% tienen un índice urbano. Estas ciudades están diseminadas a lo largo de toda la cuenca, donde destacan: Ourense, Ponferrada, Lugo, Ponteareas o Tui.

Esta disparidad entre el mundo rural y urbano se ha acrecentado en la última década, ya que la población de la cuenca del Miño-Sil ha decrecido demográficamente. No obstante, ha habido varios municipios que han conseguido aumentar su población, lo que ha provocado que se acentúe este contraste. Este desequilibrio se ha debido a que las localidades que más han crecido han sido las capitales de provincia y sus municipios adyacentes. Ejemplo de ello son Ourense, Lugo, Ponferrada, Tui o Viana do Castelo.

Este crecimiento que han experimentado las ciudades, en contraposición a las poblaciones más pequeñas, es debido a la fuerte atracción económica que tienen las grandes poblaciones sobre las pequeñas, ya que en las ciudades más grandes es donde más oportunidades laborales y servicios se concentran, por lo que muchas personas optan por abandonar los pueblos pequeños para trasladarse a las ciudades.

\subsection{EVOLUCIÓN DE LA POBLACIÓN ACTUAL 2001-2011}

La cuenca internacional del río Miño-Sil ha tenido un descenso poblacional del $1,01 \%$ lo que ha supuesto una pérdida de 11.086 personas. No obstante, este decrecimiento ha sido desigual a ambos lados de la frontera, ya que en la demarcación española ha sido de 5.647 habitantes $(-0,67 \%)$, mientras que en la parte portuguesa ha sufrido un descenso 5.439 habitantes $(-2,17 \%)$ (Figura 4$)$

Las poblaciones más grandes de la cuenca son Ourense, Lugo y Viana do Castelo, reuniendo entre ellas a una población de 294.734 habitantes, lo que supone el $27,17 \%$ del total.

Por demarcaciones el descenso registrado en la parte española es de un $-0,67 \%$. No obstante, los municipios que más han descendido demográficamente son: Barjas ($35,97 \%)$, A Merca (-30,09\%) o Benuza (-29,39\%), entre otros. Por el contrario, las localidades que mayor crecimiento han experimentado han sido: Barbadás $(44,98 \%)$, Salceda de Caselas $(36,48 \%)$ o Camponaraya $(32,41 \%)$, entre otros.

Las tres ciudades más pobladas de la parte española son: Ourense, Lugo y Ponferrada, reuniendo entre ambas a una población de 247.517 habitantes, lo que supone el $29,47 \%$ de toda la demarcación. 
Figura 4. Mapa de la evolución de la población para el periodo 2001- 2011 de las poblaciones ubicadas en la cuenca internacional del río Miño-Sil.

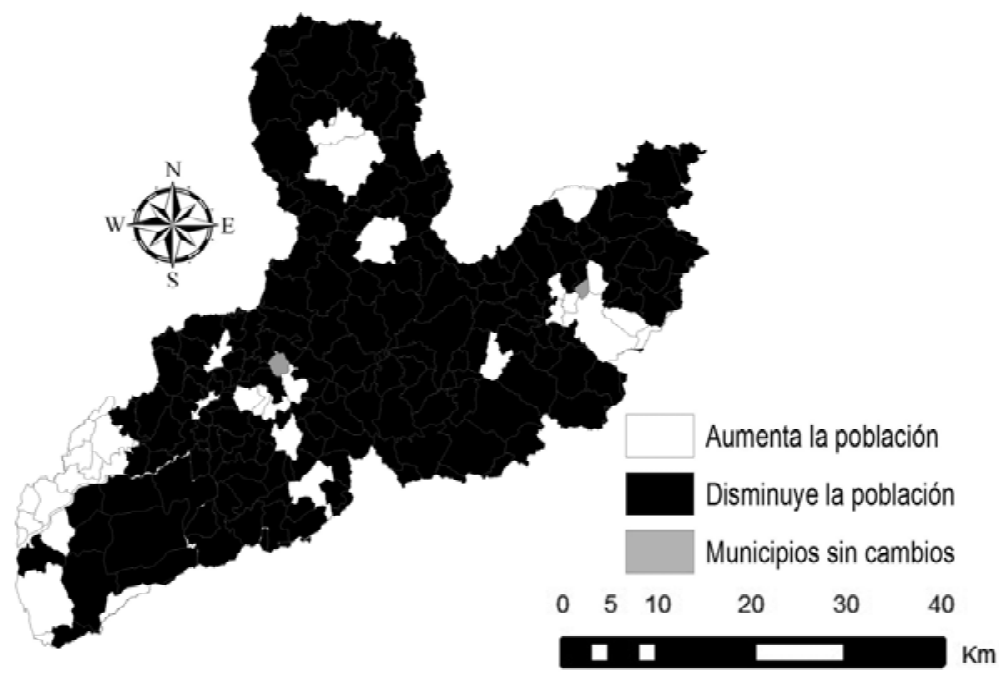

Fuente: Elaboración propia, a partir de los dados del INE-ES-PT. 2001-2011 y los dados del CNIG (Centro nacional de Información Geográfica).

En la parte portuguesa la variación de la población ha sido descendente, ya que ha habido una caída del $-2,17 \%$. No obstante, no todos los municipios han experimentado una caída en su población, pues algunos de ellos han conseguido aumentarla, por ejemplo Vila Nova de Cerveira con un crecimiento del $4,53 \%$ y Viada do Castelo con tan sólo un crecimiento del 0,11\%. En el lado opuesto se encuentran los que han sufrido un descenso demográfico como son Melgaço $(-7,83 \%)$, Arcos de Valdevez (-7,73\%) y Ponte de Barca (-6,57\%).

Las tres ciudades más pobladas de la demarcación portuguesa son: Viana do Castelo, Ponte de Lima y Arcos de Valdevez reuniendo una población total de 150.070 habitantes, lo que supone el $63,34 \%$ de la población portuguesa residente en la cuenca. Este porcentaje es mucho mayor que el de la parte española, donde las tres ciudades más pobladas representan el $29,47 \%$. 


\subsection{ESTRUCTURA DE LA POBLACIÓN}

En la pirámide de población de la cuenca internacional del río Miño-Sil del año 2011 se observa que hay un predominio del sexo femenino (50000 mujeres más que hombres), con un ratio de masculinidad del $91,16 \%{ }^{8}$ (Figura 5).

Figura 5. Pirámide poblacional de la cuenca internacional del río Miño-Sil (2011).

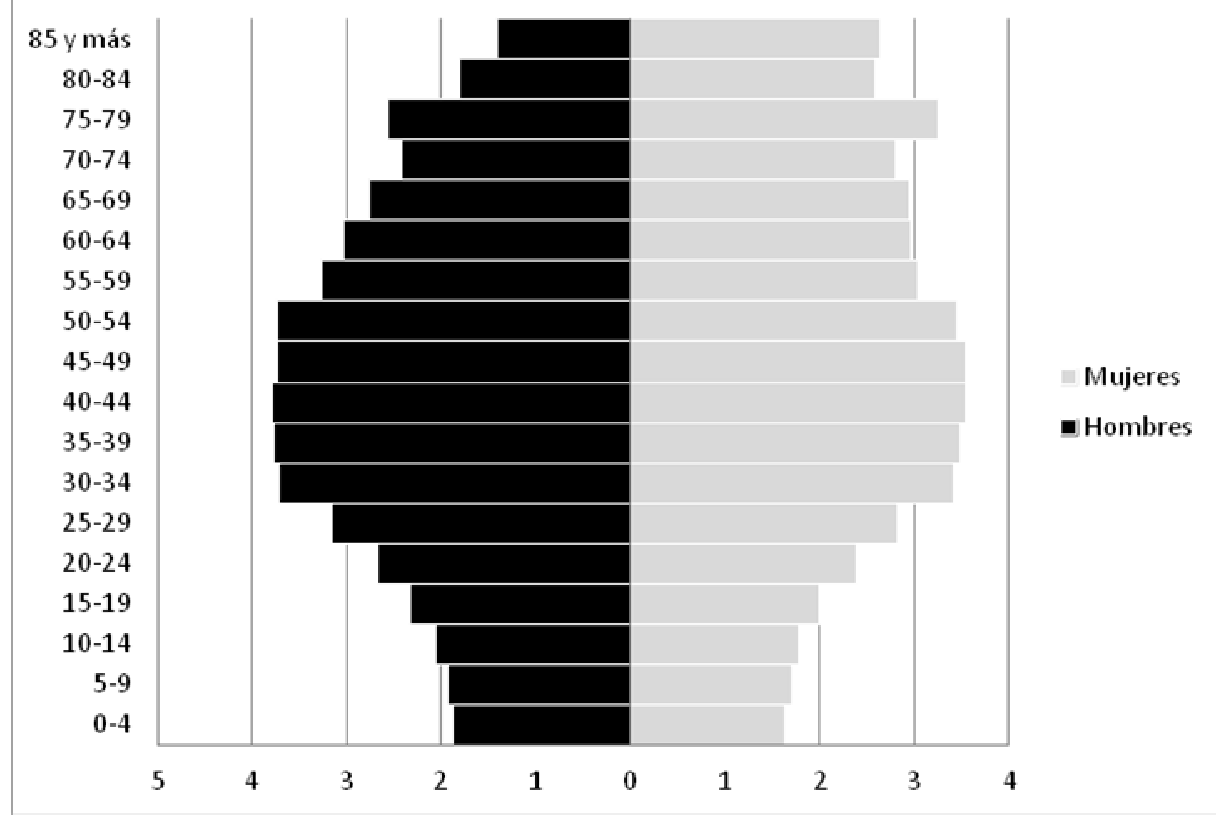

Fuente: Elaboración propia, a partir de los datos del INE-ES-PT. 2001-2011.

No obstante, la superioridad de los efectivos femeninos no se observa en toda la pirámide. En los primeros años hay más efectivos masculinos, ya que nacen más varones que mujeres (106 niños por cada 100 niñas) como se puede observar en la mayor longitud de la barra inferior de la pirámide en el lado de los varones. A partir de ahí los efectivos se van equilibrando de forma paulatina a medida que se asciende en los grupos de edad de la pirámide poblacional. Se observa que es a partir de los treinta cuatro años las mujeres empiezan a predominar en todas las cohortes de la

${ }^{8}$ Se define como número de varones por cada 100 mujeres residentes en la cuenca Internacional del río Miño-Sil a 1 de enero del año 2011. RM = Varones/Mujeres*100 
pirámide, llegando incluso en la última cohorte $(>85)$ a duplicar el número de mujeres frente al de los hombres. Esto se debe a la mayor esperanza de vida femenina: 84 años frente a los 78 de los hombres. La mayor esperanza de vida de las mujeres se debe a la sobremortalidad masculina por razones de tipo biológico, laboral y social (estilo de vida más expuesto a los factores de riesgo) (Figura 5) (INE 2011).

La pirámide de población de la cuenca presenta una clara forma de bulbo, con una base que se estrecha, indicativa de cohortes cada vez menos numerosas, debido a la baja natalidad y una cumbre con escalones más amplios que muestran un importante volumen de población adulta, debido a la mayor esperanza de vida. Esto nos permite afirmar que estamos ante una población envejecida, característica de las sociedades desarrolladas.

En el análisis por grupos de edades encontramos una amplia presencia de efectivos en el grupo de población mayor a 65 años y más. En este grupo se observa un claro predomino de los efectivos femeninos $(58,78 \%$ del total de población mayor a 65 años) frente al grupo masculino, debido a la mayor longevidad de la mujer y a la alta emigración al exterior que fue mayoritariamente de población masculina y que afectó muy significativamente a las poblaciones de la cuenca internacional del río del MiñoSil (INE 2001).

En este grupo hay un entrante en las edades comprendidas entre los 70 y 74 años (nacidos entre 1937 y 1941) que afecta a los dos sexos: corresponde a los "no nacidos" durante la Guerra Civil Española. Este hecho se vio agravado porque los adultos que debían procrear en ese momento también eran escasos ("generación hueca" de los nacidos en menor cantidad durante las guerras de finales del siglo XIX y de la epidemia de gripe de 1918).

Esta "generación hueca" se prolonga en los siguientes escalones (hasta los 55-59 años) debido a la escasa natalidad en los años de posguerra por la penosa situación que siguió a la Guerra Civil (bloqueo internacional al régimen de Franco, autarquía económica, desabastecimiento...) y a la emigración exterior en su etapa de mayor intensidad (1960-1973) (INE 2001).

El grueso de la población de la cuenca $(63,71 \%)$ se encuentra dentro de la población adulta (15-64 años). En este grupo, los aspectos demográficos más significativos serían, primero, el aumento de la natalidad debido al llamado baby boom de las décadas sesenta y principio de los setenta. Las cohortes nacidas entre 1957-1977 (34-54 años) corresponden con el boom de la natalidad. Este fenómeno estuvo motivado por el desarrollo económico que vivió el país desde finales de la década de los cincuenta hasta mediados de los setenta. El segundo aspecto demográfico a considerar en el grupo de población adulta es el retranqueo que comienza a sufrir la pirámide a partir de la cohorte de edad 30- 34 años (1997), cuando la longitud de las barras comienzan a reducirse debido a la disminución de la fecundidad, que fue consecuencia de las importantes transformaciones políticas, sociales y económicas que se produjeron tanto en la sociedad española como en la portuguesa a partir de 1975 y 1974 respectivamente (INE 2011). 
El grupo de jóvenes (0-14 años) es muy reducido, ya que sólo contiene el 10,94\% de la población de la cuenca, debido a la continua disminución de la fecundidad antes mencionada, por lo que desde 1977 la población joven ha ido reduciéndose cohorte a cohorte, lo que tiene una consecuencia directa en el envejecimiento de la población y pone en peligro el reemplazo generacional.

En definitiva, la pirámide correspondiente a la población de la cuenca internacional del río Miño-Sil nos presenta una población envejecida, propia de una sociedad desarrollada. Las causas de este envejecimiento de la población hay que buscarlas en una natalidad muy baja y en una esperanza de vida elevada. La primera es fruto de los cambios de mentalidad (secularización de la sociedad, transformación de las costumbres familiares, incorporación de la mujer al mundo laboral, disponibilidad de medios anticonceptivos, entre otros), de la crisis económica que afectó al mundo desarrollado desde 1973 (crisis del Petróleo), el desarrollo cultural (prolongación de los estudios) y el aumento del nivel de vida de la población desde mediados de los setenta traen consigo una considerable reducción de la fecundidad.

El envejecimiento de la población, al aumentar la tasa de dependencia, puede plantear problemas a medio y largo plazo de viabilidad de los actuales sistemas de protección social, especialmente en lo que se refiere a las pensiones, así como en los aspectos sanitarios y asistenciales. Sin embargo, éste no es tanto un problema demográfico como económico, ya que si aumenta el empleo la tasa de dependencia puede disminuir con la llegada de jóvenes inmigrantes que aporten más ingresos al sistema, mediante las cotizaciones.

En el futuro es previsible que el envejecimiento se acentúe en la cuenca internacional del río Miño-Sil, debido a que la población comprendida entre los $55 \mathrm{y}$ 64 años (12,29\% de la población total) comenzará a jubilarse a corto y medio plazo, lo que generará dificultades si no se garantiza el relevo generacional o la llegada de nuevos pobladores.

\subsection{MODELOS PREDICTIVOS DE EVOLUCIÓN DE LA POBLACIÓN (2023)}

En el caso de mantenerse las tendencias demográficas actuales de la cuenca internacional del río Miño-Sil, la población para los próximos años se vería reducida, ya que el número de nacimientos seguiría decreciendo en los próximos años. Esto es debido a que desde 1977 (cohorte de edad 30-34 años) la población ha venido descendiendo de forma paulatina, por lo que el descenso de nacimientos vendría determinado por la reducción del número de mujeres en edad fértil, al encontrarse en esas edades aquellas generaciones de mujeres menos numerosas que nacieron durante la crisis de natalidad de la década de los ochenta y noventa.

Otra de las causas sería que para el año 2022 la esperanza de vida en los varones alcanzaría los 81,8 años y los 87 en mujeres, lo que supone una ganancia de 2,5 años y de 1,9 años respectivamente, a los valores actuales. Al mismo tiempo, la esperanza de vida para las personas que tengan 65 años se incrementaría hasta en 20,2 años más en los varones y 24,1 años en las mujeres para el 2022, lo que provocaría un sobreenvejecimiento de la población mayor. Este hecho tendrá como consecuencia que la 
tasa de mortalidad crezca, debido a que cada vez habrá más población mayor en los grupos de edad más elevados (2014).

Una de las posibles consecuencias es que para el año 2023 habría más defunciones que nacimientos, lo que provocaría un saldo vegetativo negativo, debido fundamentalmente al descenso de la natalidad. Por edades, la población para el 2023 se verá reducida en los tramos de edad entre 20 y 49 años, mientras que la población se incrementaría en la mitad superior de la pirámide de población. De hecho, todos los grupos de edad a partir de los 50 años experimentarían un crecimiento de efectivos.

Las consecuencias del envejecimiento de la población comportarán un reto para la sociedad futura, ya que los cambios demográficos que se están produciendo van a requerir reformas sociales, económicas y políticas que el proceso de envejecimiento exija. Este hecho puede tener un afecto directo en el aumento de los gastos sanitarios, prestaciones sociales, y discapacidad, debido al mayor número de personas jubiladas y menor población activa, ya que para el 2023 está estimado que haya un trabajador por cada jubilado lo que podría poner en peligro la sostenibilidad del sistema.

Esta situación se puede revertir siempre y cuando el modelo demográfico cambie, por lo que se deben incentivar políticas para aumentar la natalidad, que provocaría un descenso en la tasa de envejecimiento. No obstante, este hecho debe estar acompañado de un mayor desarrollo económico que provoque un aumento de la inmigración y así rejuvenecer la estructura de la población en la cuenca.

\section{CONCLUSIONES}

En la cuenca internacional del río Miño-Sil viven un total de 1.084 .967 personas distribuidas en 191 municipios, de los cuales, en torno al 51,31\% de ellos tiene una población inferior a los 2000 habitantes, aunque en estos municipios solo viven el $10,15 \%$ de la población, por el contrario, en los municipios de más de 10.000 habitantes viven el 63,30\% del total de población. Por lo que se puede concluir que la cuenca internacional del río Miño-Sil presenta una distribución espacial de la población muy dispersa en la que se alterna desiertos poblacionales con concentraciones medias y altas de población en los núcleos de mayor relevancia del territorio correspondientes a las cabeceras comarcales, capitales regiones y de provincia.

Del análisis poblacional de la última década se extrae que los municipios que integran la cuenca internacional del río Miño-Sil tienen un índice de ruralidad predominante, pero hay que destacar otros índices como el rural intermedio y el urbano que tienen un importante peso. Esta disparidad entre el mundo rural y urbano se ha acrecentado en la última década, ya que a pesar de que en conjunto la población ha descendido demográficamente no lo ha hecho de forma equitativa, lo que ha provocado que se acentúe este contraste demográfico. Este desequilibrio se debe a que las localidades que más han crecido en la década analizada han sido las capitales de provincia o regionales y sus municipios adyacentes. 
La pirámide poblacional de la cuenca internacional del río Miño-Sil representa a una población envejecida, propia de una sociedad desarrollada, debido a la baja natalidad y al aumento de la esperanza de vida. De mantenerse las tendencias demográficas actuales, se pondría en peligro el sistema actual de protección social, especialmente en lo que se refiere a la pensiones, ya que para el 2023 se ha estimado que haya un trabajador por cada jubilado lo que pondría en peligro la sostenibilidad del sistema.

\section{REFLEXIONES ${ }^{9}$}

Las peculiaridades de la distribución espacial de la población ubicada en la cuenca internacional del río Miño-Sil constituyen, mayoritariamente, condiciones desfavorables para abordar con éxito un proceso solvente de ordenación del territorio. Nos encontramos con un sistema de poblamiento que guarda poca relación con la estructura económica actual y cuya dispersión y debilidad demográfica complica la prestación de servicios y dotación de infraestructuras y el mantenimiento de una mínima actividad económica.

Ante tal realidad, la estructuración del territorio de la cuenca internacional del río Miño-Sil ha de hallar un punto de equilibrio entre la conservación de los espacios rurales (depositarios de un gran poso eco-cultural) y la toma de decisiones que supongan cambios más o menos significativos tanto a nivel cualitativo como cuantitativo.

Todo el proceso debe descansar, inevitablemente, en la potenciación de la red de núcleos cabecera de área funcional, intentando romper la atonía demográfica y económica que los viene caracterizando y haciendo de ellos centros capaces de prestar servicios públicos intermedios a su zona de influencia y a la vez de servirse de éstas en cuanto soporte de actividades comerciales.

La superación de las dificultades para conseguir un territorio equilibrado desde el punto de vista de la distribución de la población, refiriendo el concepto de equilibrio a un nuevo modelo de ruralidad cuya definición integral no debería demorarse, requerirá de un amplio consenso sociopolítico basado en la solidaridad y consciente a la vez de la unidad del espacio regional, con unos flujos de posesión/mantenimiento, uso de éste entre los ámbitos rural y urbano caracteriza por la fluidez y la renuncia al patrimonialismo excluyente (López y Prieto, 1999).

${ }^{9}$ Queremos agradecer la inestimable ayuda recibida por parte del Licenciado en Geografía y Ordenación del Territorio David Hernández. 


\section{BIBLIOGRAFÍA}

AGARWAL, S., RAHMAN, y S., ERRINGTON, A. (2009). Measuring the determinants of relative economic performance of rural areas. Journal of Rural Studies $n^{\circ} 25,309-321$.

BRADEN, S., y MAYO, M. (1999). Culture, community development and representation. Community Development Journal no 34, 191-204.

BRENNAN, M.A., FLINT, C.G., y LULOFF, A. E. (2008). Bringing Together Local Culture and Rural Development: Findings from Ireland, Pennsylvania and Alaska. Sociologia Ruralis 2008.

BRIDGER, J.C., y LULOFF, A.E. (1999). Toward an interactional approach to sustainable community development. Journal of Rural Studies $\mathrm{n}^{\circ} 15,377-387$.

COPUS, A.K., COURTNEY, P., DAX, T., MEREDITH, D., NOGUERA, J., SHUCKSMITH, M., y TALBOT, H. (2011). European Development Opportunities for Rural Areas (EDORA). Applied Research 2013/1/2. Final Report, ESPON 2013 Programme, Brussels.

CONFEDERACIÓN HIDROGRÁFICA DEL MIÑO-SIL (2014).

http://www.chminosil.es/es/chms/planificacionhidrologica/planhidrologico [consulta: 1 de Septiembre de 2014]

COURTNEY, P., HILL, G., y ROBERTS, D. (2006). The role of natural heritage in rural development: an analysis of economic linkages in Scotland. Journal of Rural Studies vol. $4 \mathrm{n}^{\mathrm{o}} 22,469-484$

EUROPEAN COMMISSION, (2010a). Investing in Europe's Future: Fifth Report on Economic, Social and Territorial Cohesion. European Commission, Brussels.

EUROPEAN COMMISSION, (2010b). Study on Employment, Growth and Innovation in Rural Areas (SEGIRA). European Commission, Brussels.

EUROPEAN COMMISSION, (2011). Cohesion Policy 2014-2020: Investing in Job and Growth. European Commission, Brussels.

HOGGART, K., BULLER, H., y BLACK, R. (1995). Rural Europe. Identity and Change. Arnold, London.

INE (2001). Censos de población y viviendas 2001. Disponible en http://www.ine.es/censo2001/

INE (2011). Censos de población y viviendas 2011. Disponible en http://www.ine.es/censos2011_datos/cen11_datos_inicio.htm.

INE (2014). Proyección $\mathrm{de}^{-}$población ${ }^{-}$a corto plazo. Disponible en http://www.ine.es/prensa/np813.pdf

JOHANSEN, P. H., y NIELSEN, N. C. (2012). Bridging between the regional degree and the community approaches to rurality- A suggestion for a definition of rurality for everyday use. Land Use Policy n ${ }^{\circ} 29,781-788$.

KAHILA, P., NEMES, G. y HIGH, C. (2009). Institutional capacity. EDORA Project, Working Paper 7 (Annex1). ESPON \& UHI Millennium Institute, Luxembourg.

LANGLAIS, R., y TEPECIK D.A. (2009). Climate Change. EDORA Project, Working Paper 8 (Annex 1). ESPON \& UHI Millennium Institute, Luxembourg. 
LEE, J., ÁRnASON, A., NightingAle, A., y SHUCKSMITH, M. (2005). Networking: social capital and identities in European rural development. Sociologia Ruralis $\mathrm{n}^{\circ} 45$.

LINDENBOIM, J., y KENNEDY, D. (2003). Continuidad y cambios en la dinámica urbana de Argentina. En VII Jornadas Aepa. Tafí del Valle. Tucumán.

LÓPEZ, L., y PRIETO, I. (1999). Evolución demográfica reciente y ordenación del territorio en Castilla y León. Revista de investigación económica y social de Castilla y León, $\mathrm{n}^{\mathrm{o}}$ 1, 87-102.

MUÑOZ, M.A. (2012). Elementos conceptuales y metodológicos para una caracterización de la ruralidad en Medellín, Colombia caso corregimiento de Altavista. Tesis de Grado. Universidad Nacional de Colombia.

NELSON, R.R., y SAMPAT, B.N. (2001). Making sense of institutions as a factor shaping economic performance. Journal of Economic Behavior and Organization. Vol. $1 \mathrm{n}^{\circ} 44,31-54$.

ONU (2008). Principles and Recommendations for Population and Housing Censuses. Revision 2. Department of Economic and Social Affairs Statistics Division, Statistical papers, Series M No. 67/Rev.2. ONU: New York. Disponible en:

http://unstats.un.org/unsd/demographic/standmeth/principles/Series_M67Rev2en.pdf

PÉREZ, E. (2001). Hacia una nueva visión de lo rural. En N. Giarracca (Comp.), ¿Una nueva ruralidad en América Latina? (pp. 17-29). Buenos Aires, Argentina: Consejo Latinoamericano de Ciencias Sociales/CLACSO.

PRIETO, M.B. (2011). Cambios y continuidades del sistema de asentamiento de la población en el sudoeste bonaerense. Huellas, ${ }^{\circ} 15,329-573$.

PUTNAM, R. (1993). Making Democracy Work: Civic Traditions in Modern Italy. Princeton University Press, New Jersey.

REQUES-VELASCO, P., y RODRÍGUEZ, V. (1996). Prospectivas demográficas y territoriales. Treballs de la Societat Catalana de Geografia, Vol. $11 \mathrm{n}^{\circ} 41$.

RIMISP, 2007. Rural Territorial Dynamics: A Research-based Policy Advice and Capacity-Development Program for Rural Economic Growth, Social Inclusion and Sound Environmental Governance. Latin American Center for Rural Development, Santiago de Chile.

SÁNCHEZ-ZAMORA, P., GALLARDO-COBOS, R., y CEÑA-DELGADO, F. (2014). Rural areas face the economic crisis: Analyzing the determinants of successful territorial dynamics. Journal of Rural Studies $n^{\circ} 35,11-25$.

SERRA, P., VERA, A., TULLA, A.F., y SALVATI, L. (2014). Beyond urban-rural dichotomy: Exploring socioeconomic and land-use processes of change in Spain (1991-2011). Applied Geography no 55, 71-81.

STELlMES, M., RÖDER, A., UDELHOVEN, T., HILL, J. (2013). Mapping syndromes of land change in Spain with remote sensing time series, demographic and climatic data. Land Use Policy $\mathrm{n}^{\mathrm{o}}$ 30, 685-702.

SVENDSEN, G.L.H., SØRENSEN, J.F.L. (2007). There's more to the picture than meets the eye: measuring tangible and intangible capital in two marginal communities in rural Denmark. Journal of Rural Studies no $23,453-471$. 
TERLUIN, I., y POST, J.H. (2000). Employment Dynamics in Rural Europe. CABI Publishing, Oxon.

WHITELEY, P. (2000). Economic growth and social capital. Political Studies. Vol. 3 $\mathrm{n}^{\circ} 48,443-466$.

ZAMUDIO, F.J., CORONA, A.L., y IRAÍS D. (2008). Un índice de ruralidad para México. Espiral, estudios sobre estado y sociedad, Vol 14 nº 42, 179-214. 